\title{
DESARROLLO Y EJERCICIO DE LOS DERECHOS EN SALUD SEXUAL Y REPRODUCTIVA
}

\author{
DEVELOPMENT AND EXERCISE OF RIGHTS IN SEXUAL AND REPRODUCTIVE HEALTH
}

\author{
Oscar Ugarte-Ubilluz ${ }^{1, a}$
}

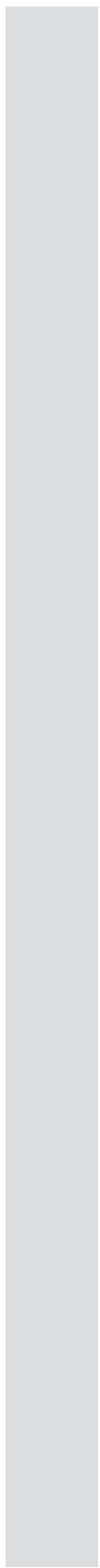

Durante décadas, el concepto de salud reproductiva estuvo circunscrito en el país, y en el nivel internacional, a una visión de la planificación familiar como forma de reducir el crecimiento poblacional y, por esa vía, luchar contra la pobreza. Sin embargo, a partir de la Conferencia Internacional de Población y Desarrollo, realizada en El Cairo en el año 1994, el concepto se amplió a salud sexual y reproductiva con una visión más integral vinculada al desarrollo, a la promoción de valores y a la defensa de los derechos humanos ${ }^{(1)}$.

Durante los primeros setenta años del siglo XX, los países y los organismos internacionales se preocupaban principalmente por las consecuencias económicas y sociales del rápido crecimiento poblacional de la época. Esto ha sido más intenso en América Latina en las décadas del 60 y 70 cuando los avances en la reducción de la mortalidad general configuraron la denominada "explosión demográfica", como un problema económico y social. Esta preocupación se expresó en la gran mayoría de países participantes en la Conferencia Internacional de Población de Bucarest, en $1974^{(2)}$. Como consecuencia de ello se adoptaron recomendaciones para establecer fuertes programas de planificación familiar, orientados fundamentalmente a reducir las elevadas tasas de fecundidad de la mayoría de países en vías de desarrollo, utilizando masivamente diferentes métodos de planificación familiar, entre ellos los métodos hormonales cuyo uso desde la década de los 60 se había popularizado con enorme éxito.

En la Conferencia Internacional de Población de $1984^{(3)}$, realizada en México, se mantuvo ese criterio aunque fuertemente vinculado también con el concepto de promoción de la salud, en el marco de la atención primaria de salud aprobada por la Organización Mundial de Salud (OMS) en la Conferencia Mundial de Alma Ata en $1978{ }^{(4)}$. En el Perú, en los años 80, la normatividad estuvo marcada por esa orientación predominante a nivel internacional. En ese período, los programas de planificación familiar se articularon estrechamente con los programas materno e infantil, cuyos objetivos eran reducir la morbimortalidad materna e infantil. Así, se constituyeron como partes de un mismo programa de salud materno-infantil y de planificación familiar.

Pero, la Conferencia Internacional sobre Población y Desarrollo (CIPD) de 1994, celebrada en EI Cairo, marcó un nuevo hito, porque los gobiernos ahí reunidos reconocieron internacionalmente los derechos reproductivos contenidos en documentos sobre derechos humanos, y declararon que la salud y los derechos sexuales y reproductivos son derechos humanos fundamentales para el desarrollo (1). Se adoptó un nuevo concepto, más amplio, de salud reproductiva y derechos reproductivos, incluyendo la planificación familiar y la salud sexual como parte de la salud integral de las personas y de la lucha por el desarrollo económico y social. Por eso no es casual que la Conferencia de El Cairo fuese sobre población y desarrollo, a diferencia de las cuatro primeras conferencias internacionales (Roma 1954, Belgrado 1965, Bucarest 1974 y México 1984) que solo fueron sobre población.

El cambio conceptual en EI Cairo incluye que la salud reproductiva es parte de los derechos fundamentales de las personas, pero también que el derecho a la sexualidad y la salud sexual es independiente de la reproducción y, por tanto, que las personas tienen todo el derecho de ejercer plenamente su sexualidad, sin necesidad de la reproducción, si así lo desean.

Sistema Metropolitano de La Solidaridad (SISOL). Lima, Perú.

Médico, magister en Gestión de Políticas Públicas, ex ministro de salud.

Recibido: 04-09-13 Aprobado: 04-09-13

Citar como: Ugarte-Ubilluz O. Desarrollo y ejercicio de los derechos en salud sexual y reproductiva. Rev Peru Med Exp Salud Pública. 2013; 30(3):376-8. 
En este contexto, el nuevo diseño de los servicios introdujo cambios importantes: de ser parte de un imperativo demográfico se convirtió en un enfoque de derechos de las personas; de ser servicios aislados se introdujo una visión integral; de tener su eje en los médicos y servidores de salud pasó a tener su eje en las personas; de estar focalizados en las mujeres en edad fértil se amplió a todas las mujeres y hombres en las diferentes etapas de la vida; de propiciar una actitud pasiva de los usuarios se procuró una actitud ampliamente participativa en el ejercicio de los derechos en salud sexual y reproductiva, pero también en el reconocimiento de las responsabilidades de todos los ciudadanos en el libre ejercicio de su sexualidad que conllevan esos derechos.

En síntesis, a partir de la Conferencia Internacional de Población y Desarrollo, puede decirse que el concepto de salud sexual y reproductiva evoluciona de un enfoque tradicional de planificación familiar a una dimensión amplia de promoción de los derechos sexuales y reproductivos. La salud sexual y reproductiva fue definida por consenso en la CIPD de El Cairo 1994 como: Un estado general de bienestar físico, mental y social, y no como la mera ausencia de enfermedades o dolencias, en todos los aspectos relacionados con el sistema reproductivo y sus funciones y procesos. En consecuencia, la salud reproductiva entraña la capacidad de disfrutar de una vida sexual satisfactoria y sin riesgos y de procrear, $y$ la libertad para decidir hacerlo o no hacerlo, y cuándo y con qué frecuencia. Esta última condición Ileva implícito el derecho del hombre y la mujer a obtener información de planificación de la familia de su elección, así como a otros métodos para la regulación de la fecundidad que no estén legalmente prohibidos, y acceso a métodos seguros, eficaces, asequibles y aceptables, el derecho a recibir servicios adecuados de atención de la salud que permitan los embarazos y los partos sin riesgo y den a las parejas las máximas posibilidades de tener hijos sanos ${ }^{(5)}$.

Un buen estado de salud sexual y reproductiva implica, por tanto, la capacidad de disfrutar de una vida sexual satisfactoria y sin riesgos, pero, al mismo tiempo, involucra la posibilidad de ejercer los derechos sexuales y reproductivos. Estos derechos se basan en el reconocimiento que tienen las parejas y los individuos de decidir libre y responsablemente el momento del matrimonio, el número y espaciamiento de los hijos, a disponer de información y de los medios para acceder a ella sin ningún tipo de discriminación de sexo, edad, etnia, clase, orientación sexual o estado civil de la persona, y teniendo en cuenta sus necesidades específicas de acuerdo con su ciclo vital ${ }^{(5)}$.
La IV Conferencia Mundial sobre la Mujer, realizada en Beijing el año 1995, permitió ampliar mucho más la dimensión de la salud sexual y reproductiva, no solo como parte de los derechos humanos en general reconocidos en la Conferencia de El Cairo, sino como parte del derecho específicamente de las mujeres a decidir sobre su salud sexual y reproductiva. Es por ello que en la segunda mitad de los 90, las políticas y programas se definieron bajo la perspectiva de género y se documentó la necesidad de integrar en la salud pública aspectos del desarrollo humano como la educación y la nutrición, entre otros: Hoy día, la SSR se refiere [en el plano personal].... a un continuo que empieza antes de la concepción, incluye la educación a temprana edad, abarca el desarrollo del adolescente y pasa por el período de la fecundidad y la reproducción hasta que, en la mujer desemboca en la menopausia y el climaterio y en el hombre en la andropausia... [en el plano colectivo]... es parte integrante del desarrollo sostenible de un país y se basa en los derechos y deberes humanos individuales y sociales ${ }^{(6)}$.

Posteriormente, en septiembre de 2000, la Declaración del Milenio ${ }^{(7)}$, adoptada por 187 países, llevó a la definición de los Objetivos de Desarrollo del Milenio (ODM) y de metas asociadas, las cuales son comúnmente aceptadas como un marco para monitorear el progreso hacia el desarrollo. De los ocho ODM definidos en el 2000, el quinto se relaciona con la salud materna, donde se plantea la meta de reducir en $75 \%$ la tasa de mortalidad materna entre 1990 y 2015. Sin embargo, a pesar de la importancia dada a la SSR en la CIPD, esta no estuvo incluida inicialmente dentro de las 18 metas asociadas a los ocho objetivos definidos en el 2000.

Recién en octubre de 2006, la Asamblea General de las Naciones Unidas acordó incluir una nueva meta al quinto objetivo del milenio: Acceso universal para el 2015 del rango más amplio posible de métodos seguros y efectivos de planificación familiar, incluyendo métodos de barrera, y acceso a los servicios de salud reproductiva: cuidado obstétrico, prevención y manejo de infecciones reproductivas incluyendo infecciones de transmisión sexual ${ }^{(6)}$.

De esta forma, con la enmienda a la quinta meta de los ODM, se ratifica el compromiso internacional con la SSR logrado en 1994 en la CIPD, y se obliga a los países a incorporar, en el marco de las reformas del sector salud, políticas que promuevan y garanticen el acceso a los servicios de salud sexual y reproductiva.

Al cumplirse en el 2014 veinte años de la Conferencia Internacional de Población y Desarrollo de El Cairo 94, y en el 2015 veinte años de la Conferencia Internacional 
sobre la Mujer de Beijing 95, le corresponde al Perú hacer una evaluación de los avances y dificultades que puedan haber tenido las orientaciones y políticas definidas dos décadas atrás y proponer las alternativas que correspondan.

El presente número de la Revista Peruana de Medicina Experimental y Salud Pública del Instituto Nacional de Salud quiere contribuir en esa tarea y ha convocado a un grupo de profesionales especializados para que aporten desde diversos aspectos de esta amplia problemática.

La Mag. Susana Galdos Silva, integrante de la delegación peruana que participó en El Cairo 94 y Beijing 95, en el tema de "Derechos en salud sexual y reproductiva" hace un interesante recuento del contexto en el cual se desarrollaron dichos eventos y de la participación de Perú. El Dr. Miguel Gutiérrez, en "Planificación familiar, herramienta básica en SSR" evalúa la importancia de la planificación familiar en el ejercicio de los derechos en salud sexual y reproductiva en las últimas décadas en el país, y los avances producidos en ese período. El Dr. Eduardo Pretell, en "Política de anticoncepción oral de emergencia" describe el contexto de la aprobación de la política de anticoncepción oral de emergencia en el año 2001, como avances en las políticas en defensa de los derechos en salud sexual y reproductiva; y narra su experiencia como ministro de salud en ese período. La Mg. Susana Chávez, en "El Aborto terapéutico en el Perú”, aborda este importante, aunque controversial, tema autorizado por el Código Penal desde el año 1924, a partir del marco de derechos y de la experiencia internacional y en nuestro propio país. La Dra. Lucy del Carpio, en "Salud de la mujer y mortalidad materna" hace un interesante y documentado enfoque de este importante objetivo de desarrollo del milenio en Perú, muestra los avances alcanzados y los desafíos hacia el futuro. La Lic. Rossina Guerrero, en "Penalización de las relaciones sexuales entre adolescentes" describe la experiencia en el Perú de lo sucedido en el marco de esta legislación aprobada en el año 2006 y recientemente suspendida por una sentencia del Tribunal Constitucional. El Dr. Daniel Aspilcueta, en "El rol del varón en la anticoncepción: su rol como pareja y como usuario" analiza uno de los nuevos temas que incorporó El Cairo 94 al definir la importancia de un enfoque integral que compromete a mujeres y hombres en los diferentes ciclos de vida. Finalmente, el Dr. Walter Mendoza de Souza y la Dra. Gracia Subiría, en "El embarazo adolescente" analizan este importante problema con alto impacto en la salud pública en el país sugiriendo orientaciones necesarias a ser incorporadas en nuestras políticas sanitarias.

Otros diversos aspectos de la salud sexual y reproductiva, no han podido ser tratados en este número como hubiésemos querido. Quedan pendientes temas importantes como el de la fertilización asistida, la diversidad sexual, la sexualidad y la explotación, o la historia de la sexualidad en el país, entre otros. Esperamos que puedan ser tratados en otra oportunidad.

Nuestro profundo agradecimiento a los autores cuyos trabajos se presentan en este número y nuestro compromiso de continuar contribuyendo en la promoción y defensa de los derechos en salud sexual y reproductiva, como parte de los derechos fundamentales de la persona.

\section{REFERENCIAS BIBLIOGRÁFICAS}

1. Galdos S. La conferencia de El Cairo y la afirmación de los derechos sexuales y reproductivos, como base para la salud sexual y reproductiva. Rev Per Med Exp y Salud Publica. 2013;30(3): 455-60.

2. United Nations. World Population Plan of Action [Internet]. New York: United Nations [citado el 12 de febrero de 2013]. Disponible en: http://www. un.org/popin/icpd/conference/bkg/ wppa.html

3. Brown GF. United Nations International Conference on Population, Mexico City, 6-13 August 1984. Studies in Family Planning. 1984;15(6):296-302.
4. Tejada de Rivero DA. Lo que es la atención primaria de la salud: algunas consideraciones a casi treinta y cinco años de Alma-Ata. Rev Peru Med Exp Salud Publica. 2013;30(2):283-7.

5. United Nations Population Information Network (POPIN). Informe de la Conferencia Internacional sobre la Población y el Desarrollo (El Cairo, 5 a 13 de septiembre de 1994) [Internet]. New York: POPIN [citado el 12 de febrero de 2013]. Disponible en: http://www.un.org/popin/icpd/ conference/offspa/sconf13.html

6. Flores CE, Soto VE. El estado de la salud sexual y reproductiva en América Latina y el Caribe: una visión global [Internet]. New York: Banco Interamericano de Desarrollo; 2008 [citado el 12 de febrero de 2013]. Disponible en: http://hivaidsclearinghouse.unesco. org/search/resources/santiago_ pubwp-632_esp.pdf

7. Naciones Unidas. Resolución aprobada por la Asamblea General. Declaración del Milenio [Internet]. New York: United Nations [citado el 12 de febrero de 2013]. Disponible en: http://www. un.org/spanish/milenio/ares552.pdf 